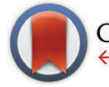

CrossMark

Cite this: J. Mater. Chem. C, 2016, 4, 11244

Received 14th October 2016, Accepted 25th October 2016

DOI: $10.1039 / c 6 t c 04453 k$

www.rsc.org/MaterialsC

\section{Fluorescent aryl naphthalene dicarboximides with large Stokes shifts and strong solvatochromism controlled by dynamics and molecular geometry $\dagger$}

\author{
Robert Greiner, Thorben Schlücker, + Dominik Zgela and Heinz Langhals*
}

\begin{abstract}
A series of highly fluorescent 4-aryl substituted naphthalene dicarboximides were efficiently prepared via metal organic $\mathrm{C}-\mathrm{C}$-coupling reactions. The obtained push-pull fluorophores display a distinct positive solvatochromism of the fluorescence. These optical properties are shown to be significantly dependant on the molecular geometry. Corresponding to TICT, a twist between the donor and the acceptor moiety enhances the intramolecular charge transfer resulting in such pronounced solvatochromism. Complete orthogonalisation inhibits the fluorescence. An intentional skew arrangement leads to solvent-adjustable chromophores with high fluorescence quantum yields and Stokes shifts of more than $1.6 \mathrm{eV}$.
\end{abstract}

\section{Introduction}

peri-Naphthylcarboximides ${ }^{1}$ are well known fluorescent dyes which find broad applications like in white light-emitting diodes. ${ }^{2}$ Naphthalimides with donor groups in position 4 such as 4 -aminonaphthalene-1,8-dicarboximides ${ }^{3}$ and 4-alkoxy-naphthalene-1,8carboximides $^{4}$ are of special interest since comparably large Stokes shifts and positive solvatochromism ${ }^{5}$ of the fluorescence are observed. A photo-induced shift of electron density from the donor to the carboximide is responsible for inducing a large dipole moment. Lowering of the energy of the excited state by solvation with polar solvents causes a bathochromic shift of the fluorescence. This process corresponds to the positive solvatochromism of 4-amino- $N$-methylphthalimide applied for Zelinskii's ${ }^{6}$ solvent polarity $S$ scale. An even more pronounced solvatochromism of naphthalimides should be obtained through the introduction of extended electron rich aryl moieties.

\section{Results and discussion}

The fluorescent solvatochromism is expected to increase with the photo-induced dipole moment depending on the distance of the separated charges. Therefore, we inserted aryl groups as conjugating spacers between the donor groups and the naphthalimide acceptor moiety to achieve such a prolongation. However, little is known about such 4 -aryl naphthalimides. ${ }^{7}$ We targeted

Department of Chemistry, LMU Ludwig-Maximilians-University Munich, Butenandtstr. 5-13, 81377 Munich, Germany. E-mail: Langhals@lrz.uni-muenchen.de; Fax: +49-89-2180-77640; Tel: +49-89-2180-77699

$\dagger$ Electronic supplementary information (ESI) available. See DOI: 10.1039/ c6tc04453k

\$ This author contributed equally to this work. their synthesis by means of transition metal-mediated arylations. To obtain highly soluble dyes, we started with a condensation of readily available 4-bromonaphthalic anhydride 1 with tridecan-7amine ${ }^{8}$ giving the highly soluble key intermediate 2 (Scheme 1 ). The Suzuki cross-coupling reaction of 2 with various aryl dioxaborolanes gave the corresponding arylated derivatives $\mathbf{3 a - d}$ and $3 \mathbf{g}-\mathbf{j}$, respectively.

Substitution with the sterically hindered 2,6-dimethyl phenyl boronate failed where synthesis could be alternatively realised by the stepwise peripheral introduction of the sterically demanding

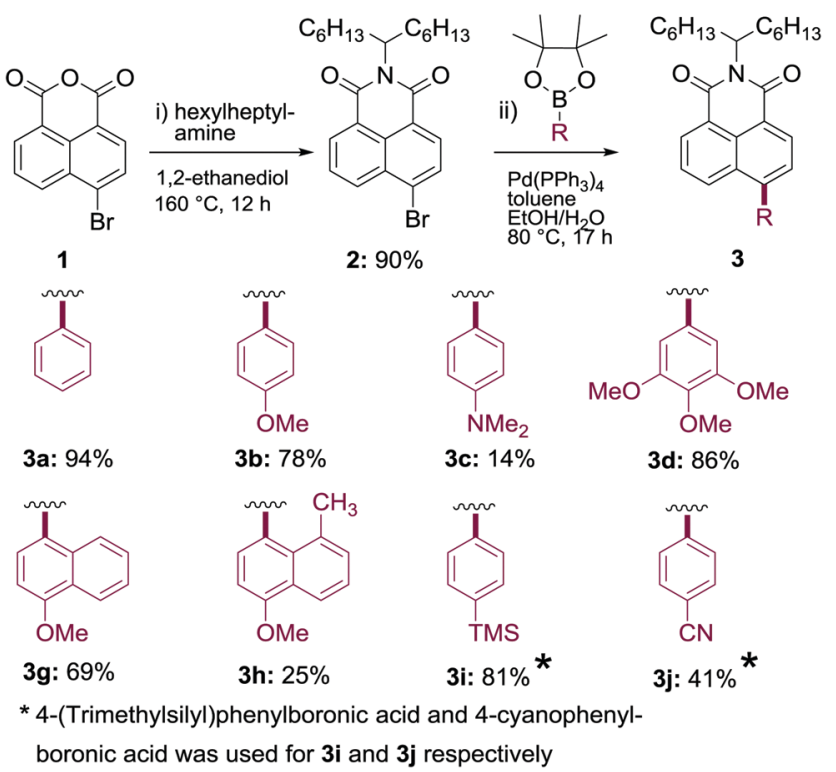

Scheme 1 Synthesis of arylated naphthalene carboximides; (i) amine condensation; (ii) Suzuki cross-coupling. 

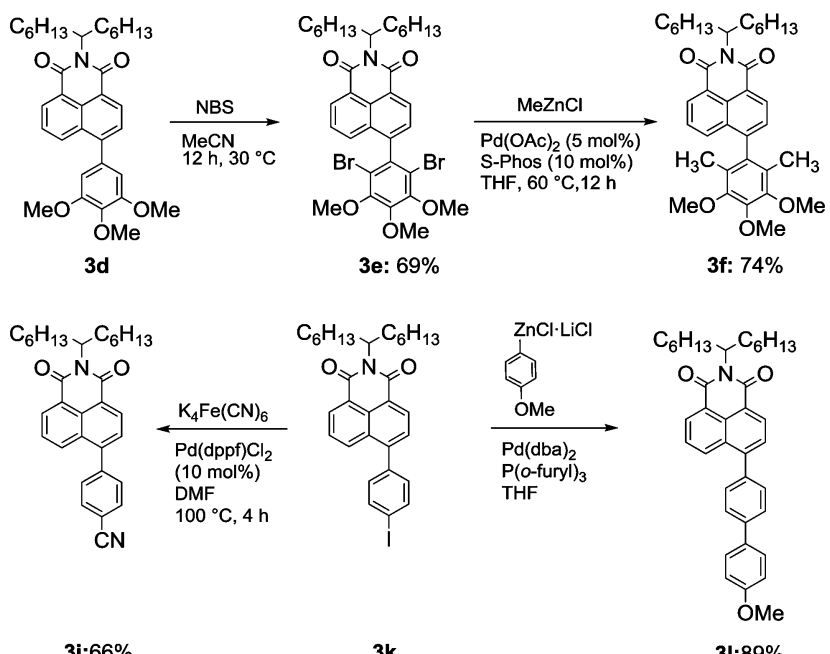

$3 \mathbf{j}: 66 \%$

3k
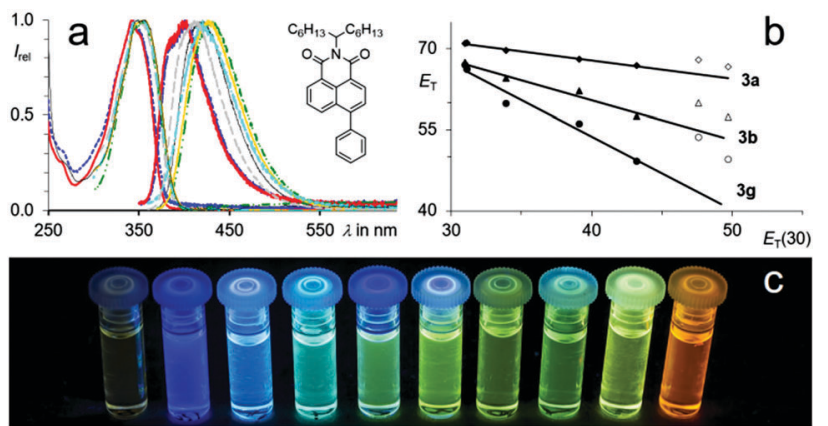

Fig. 1 (a) UV/Vis absorption (left) and fluorescence (right) spectra of $3 a$ in various solvents. From left to right: $n$-hexane (thick solid red), $n$-tetradecane (dotted blue), toluene (dashed grey), chloroform (solid black), 1-undecanol (dotted dashed turquoise) DMF (diffuse yellow), 1-butanol (double dotted dashed). (b) Linear relationship of the solvatochromism $\left(E_{T}\right)$ of $3 a$ (diamonds), $\mathbf{3} \mathbf{b}$ (triangles) and $\mathbf{3 g}$ (circles), respectively to the $E_{\mathrm{T}}(30)$ solvent polarity scale. Closed symbols: aprotic solvents; open symbols: protic solvents, neglected for regression. Scales are in $\mathrm{kcal} \mathrm{mol}^{-1}$. (c) Aryl naphthalene carboximides $\mathbf{3}$ in $\mathrm{CHCl}_{3}$ under UV-light (366 nm). From left to right: 3f, 3j, 3b, 31, 3a, 3i, $3 \mathrm{~h}, 3 \mathrm{~g}, 3 \mathrm{~d}, 3 \mathrm{c}$.
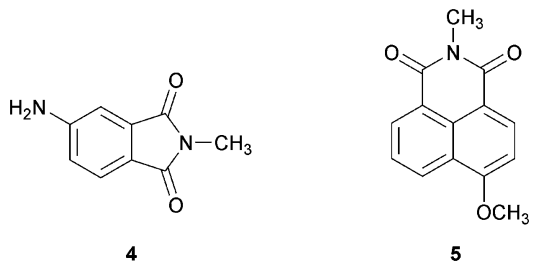

corresponding aryl iodide $\mathbf{3 k}$ in order to extend the conjugated system. A typical Negishi cross-coupling of $\mathbf{3 k}$ with $p$-anisylzinc chloride $^{10}$ (prepared from the reaction of 4 -iodoanisole with iPrMgCl-LiCl, followed by $\mathrm{ZnCl}_{2}$ ) gave the methoxy biphenyl derivative $\mathbf{3 l}$ in very good yield. Further, the cyanation of $\mathbf{3 k}$ led to the corresponding arylnaphthyl cyanide $\mathbf{3} \mathbf{j}$ in an improved yield (Scheme 2).

From UV/Vis and fluorescence measurements it can be clearly recognized that our new dyes 3 are moderately solvatochromic in absorption (Fig. S24 and S26-S34, ESI $\dagger$ ) and strongly solvatochromic in fluorescence as shown for the simple phenyl derivative 3a (Fig. 1a and Fig. S25, ESI $\dagger$ ). This indicates an optical excitation-induced increase of the dipole moment and was subject of further investigations. The molar energies of fluorescence light of various carboximides were calculated by means of eqn (1) where $\lambda_{\max }$ is the fluorescence maximum of the individual dye in the tested solvent $\left(E_{\mathrm{T}}\right.$ values ${ }^{11}$ are in $\mathrm{kcal} \mathrm{mol}^{-1}$ for comparison with previously reported values in the literature to avoid confusion; these may be multiplied by 4.2 to obtain SI units). The solvatochromism of the carboximides was analysed according to various theoretical approaches. Those of Kawski, ${ }^{12}$ Kamlet, Taft and Abboud ${ }^{13}$ or Catalán ${ }^{14}$ fitted our experimental results well. The respective analyses are found in the supporting information in Chapter 4. Furthermore, we investigated the fluorescent solvatochromism in more detail using the concepts of Brooker's $\chi_{\mathrm{R}}$ scale $^{15}$ and Dimroth and Reichardt's $E_{\mathrm{T}}(30)$ polarity scale ${ }^{11}$ which delivered the best results. The first represents mainly the polarisability of the solvent whereas the second indicates mostly the effect of dynamic solvation. The spectroscopic

Fig. 2 4-Amino-N-methylphthalimide (4) and 4-methoxy-N-methylnaphthalimide (5) as reference for fluorescence data comparison.

data of dyes 3 were compared to reported data to evaluate the solvent sensitivity of the fluorescence. The highly solvatochromic 4-amino- $N$-methylphthalimide (4) as the basis of Zelinskii's universal $S$ solvent polarity scale ${ }^{6}$ served as reference as well as the simple donor substituted 4-amino- $N$-methylnaphthalimide (5) (Fig. 2). The $E_{\mathrm{T}}$ values of $\mathbf{4}$ were calculated from literature data for various solvents. A linear free energy relation (LFER) ${ }^{16}$ of these $E_{\mathrm{T}}$ values with the $E_{\mathrm{T}}(30)$ polarity scale according to eqn (2) gave appreciably better results (correlation number $r=0.95$ for $n=14$ solvents) than with Brooker's $\chi_{\mathrm{R}}$ scale $(r=0.90$ for $n=14)$.

$$
\begin{aligned}
& E_{\mathrm{T}}=28591 / \lambda_{\text {max }} \\
& E_{\mathrm{T}}=a \cdot E_{\mathrm{T}}(30)+b
\end{aligned}
$$

Similar results were obtained for the solvatochromism of the fluorescence of 3 (Table 1). As a consequence, we conclude that the solvent effects by polar dynamic orientation of the solvent molecules dominate for the reported carboximides and agreed with the $E_{\mathrm{T}}(30)$ scale as most appropriate comparison.

We investigated the solvents $n$-tetradecane, $n$-hexane, toluene, chloroform, $N, N$-dimethyl formamide (DMF), 1-undecanol and 1-butanol for an overview of solvent effects where the two protic solvents were applied for studying the influence of hydrogen bonds. Linear correlations of the $E_{\mathrm{T}}$ values with the $E_{\mathrm{T}}(30)$ 
Table 1 Solvatochromism of the fluorescence of the carbox-imides $\mathbf{3}$, 4 and 5

\begin{tabular}{lllll}
\hline Dye & $\Phi^{a}$ & $\tau^{b}$ & $\alpha^{c}$ & $r$ \\
\hline $\mathbf{4}$ & & & -0.60 & -0.95 \\
$\mathbf{5}$ & & & -0.27 & -0.89 \\
3a & 0.78 & 3.21 & -0.34 & -0.99 \\
3b & 0.83 & 4.11 & -0.74 & -0.99 \\
3c & 0.64 & 6.99 & -1.49 & -0.96 \\
3d & 0.65 & 6.62 & -1.43 & -0.98 \\
3f & $<0.05$ & & & \\
3g & 0.39 & 4.07 & -1.36 & -0.98 \\
3h & 0.40 & 4.14 & -1.37 & -0.99 \\
3i & 0.79 & 3.08 & -0.34 & -0.99 \\
3j & 0.54 & 1.34 & -0.80 & -0.99 \\
31 & 0.67 & 2.93 & -1.33 & -0.98
\end{tabular}

Applied solvents: $n$-tetradecane, $n$-hexane, toluene, chloroform, dimethylformamide (DMF). ${ }^{a}$ Fluorescence quantum yield $\Phi$ in chloroform. ${ }^{b}$ Fluorescence lifetime $\tau$ in ns in chloroform. ${ }^{c}$ Slope $\alpha$ of the linear regression. ${ }^{d}$ Coefficient $r$ of correlation for applications of eqn (2).

values were obtained. Larger deviations to higher $E_{\mathrm{T}}$ were observed for hydrogen bond-donating solvents such as 1-butanol and 1-undecanol indicating the specific influence of such interactions; the solvent viscosity seems to have a minor influence (compare hexane with tetradecane and 1-butanol with 1-decanol) and large Stokes shifts are even observed in a solid glassy matrix of PMMA. As a consequence, the further discussion was concentrated on the non-hydrogen bond-donating solvents for better comparability between the dyes 3-5. A slope $\alpha$ of -0.60 is found for 4 -amino- $N$-methylphthalimide (dye 4, Table 1 ) and characterises the sensitivity of this highly solvatochromic fluorescent dye to polar solvent effects. In comparison, this interaction is appreciably lower for the methoxynaphthalimide $5(\alpha=-0.27)$ and indicates a smaller alteration of the molecular dipole moment with optical excitation. An extension of the conjugated system of the naphthalimide with a phenyl group in 3a increases the slope slightly to $\alpha=-0.34$ (Fig. 1b). Further introduction of a donor group into the $p$-position of the phenyl substituent to obtain $\mathbf{3 b}$ establishes a donor acceptor system between the methoxy- and the carbonyl groups and enhances the sensitivity $(\alpha=-0.74)$ to exceed the solvatochromism of 4 by far. The dimethylamino group of derivative $3 \mathbf{c}$ causes an even higher sensitivity towards solvents, however, the fluorescence quantum yield strongly decreases in polar solvents. Multiple donor groups as in 3d also display a remarkably high solvatochromism with comparably high fluorescence quantum yields; even though weak fluorescence was still observed in polar DMF. A substitution with larger aryl groups like the 4-methoxynaphthyl moiety leads to $3 \mathbf{g}$ which displays a very distinct solvatochromism $(\alpha=-1.36)$ while still exhibiting high fluorescence quantum yields in polar solvents. Further extension of the conjugated framework to the methoxybiphenyl derivative $\mathbf{3 1}$ also induces such a pronounced fluorescent solvatochromism $(\alpha=-1.33)$ exceeding that of the anisyl-substituted species $\mathbf{3 b}$.

Finally, the effect of the donor acceptor motif in $\mathbf{3 b}$ was further tested with $\mathbf{3} \mathbf{j}$ where the electron donating methoxy group was exchanged by an electron withdrawing cyano moiety. There is still a comparably high sensitivity to solvent polarity,

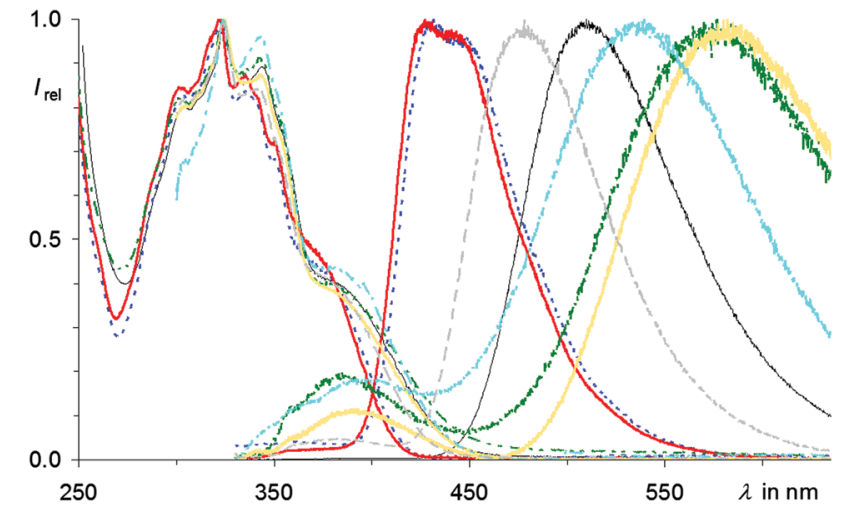

Fig. 3 UV/Vis absorption (left) and fluorescence (right) spectra of $\mathbf{3 g}$ in various solvents. From left to right: $n$-hexane (thick solid red curves), $n$-tetradecane (dotted blue curves), toluene (dashed grey curves), chloroform (thin solid black curves), 1-undecanol (dotted dashed turquoise curves) DMF (diffuse yellow curves), 1-butanol (double dotted dashed green curves).

but as expected, the effect of the donor-substituted derivatives was not reached (Table 1). The electronic properties of the 4-methoxynaphthyl derivative $3 \mathrm{~g}$ are comparable to those of compound $\mathbf{3 b}$. However, the slope parameter $\alpha$ is found to be nearly twice as much. This observation made us focussing more intensely on the geometrical arrangement of the chromophores and prompted us to investigate the influence of steric hindrance on mesomerism. In comparison, the optical properties of $\mathbf{3 g}$ (Fig. 3) and its methylated analogue $\mathbf{3 h}$ are only slightly different from each other (Fig. S35, ESI $\dagger$ ). This indicates a similar intramolecular geometry.

A skew arrangement of the aromatic systems seems to be mainly influenced by peri hydrogen atoms of the naphthalene subunits. These findings were further confirmed by quantumchemical DFT calculations (B3LYP 6-311 ${ }^{*} \mathrm{G}$ ) as shown in Table 2, Fig. 4 and Table S10, S11 (ESI $\dagger$ ). Hence, the steric influence of the methyl group in $\mathbf{3 h}$ is only subordinated (dihedral angle $77.38^{\circ}$ )

Table 2 Optimized structures and calculated dipole moments of 3 (DFT B3LYP 6-311**G)

\begin{tabular}{llllr}
\hline Dye & $\theta^{a}$ & $\theta_{\mathrm{ex}}{ }^{b}$ & Dipole $^{c}$ & Dipole $_{\mathrm{E} 1}{ }^{d}$ \\
\hline $\mathbf{3 a}$ & 57.8 & 39.64 & 5.52 & 7.75 \\
$\mathbf{3 b}$ & 55.61 & 35.86 & 6.40 & 9.82 \\
$\mathbf{3 c}$ & 51.35 & 31.43 & 9.20 & 14.86 \\
$\mathbf{3 d}$ & 57.34 & 35.67 & 6.77 & 9.22 \\
$\mathbf{3 f}$ & 87.42 & 89.36 & 6.08 & 7.22 \\
$\mathbf{3 g}$ & 71.60 & 43.34 & 6.47 & 12.21 \\
$\mathbf{3 h}$ & 75.38 & 44.94 & 6.03 & 12.20 \\
$\mathbf{3 i}$ & 57.31 & 36.39 & 6.01 & 8.53 \\
$\mathbf{3 j}$ & 57.95 & 36.57 & 0.47 & 1.66 \\
$\mathbf{3 1}$ & 56.66 & 32.24 & 6.94 & 11.26 \\
& $39.56^{e}$ & $31.92^{e}$ & & \\
$\mathbf{4}$ & & & 5.20 & 8.92 \\
$\mathbf{5}$ & & & 6.29 & 7.96
\end{tabular}

Applied solvents: $n$-tetradecane, $n$-hexane, toluene, chloroform, dimethylformamide (DMF). ${ }^{a}$ Calculated dihedral angle $\theta$ in the ground state. ${ }^{b}$ Calculated dihedral angle $\theta$ in the first electronically excited state. ${ }^{c}$ Dipole moment in the electronic ground state in Debye. ${ }^{d}$ Dipole moment in the first electronically excited state in Debye. ${ }^{e}$ Dihedral angle between phenyl moieties. 

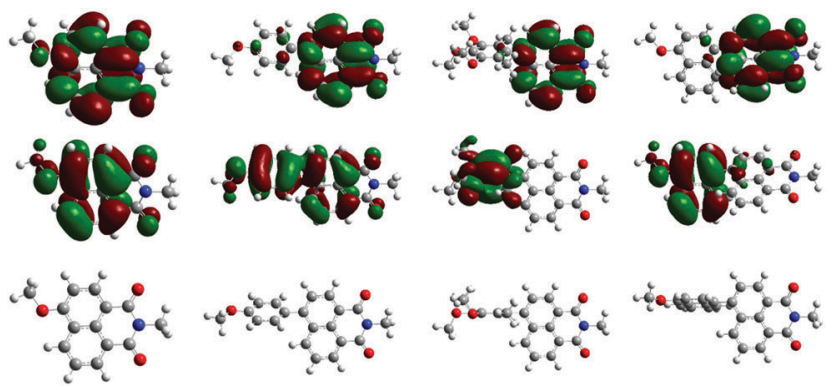

Fig. 4 Quantum chemical calculations. From bottom to top: lowest energy structures (ground state, B3LYP 6-311**G), HOMO (middle) and LUMO (top) orbitals. Left to right: $\mathbf{5}, \mathbf{3 b}, \mathbf{3 f}, \mathbf{3 g}$.

and does not affect the geometry significantly $\left(70.60^{\circ}\right.$ for $\left.3 \mathrm{~g}\right)$. Comparison of the 3,4,5-trimethoxyphenyl naphthalimides 3d $\left(57.34^{\circ}\right)$ and $3 \mathbf{f}$ exhibits much more pronounced effects. The steric repulsion of the methyl groups in $\mathbf{3 f}$ arranges the two aromatic systems statically fixed. The nearly orthogonal geometry $\left(87.42^{\circ}\right.$, Table 2 and Fig. 4) results in low fluorescence quantum yield of less than 0.05 .

A twisted geometry between the donor and the acceptor promotes charge transfer causing strong solvatochromism in fluorescence and large Stokes shifts. Moderate angles below $80^{\circ}$ preserve high fluorescence quantum yields. A complete orthogonalisation (3f) quenches fluorescence where an obviously essential residual orbital overlap is lacking. We further confirmed this concept by heating a solution of $\mathbf{3 f}$ in diethylene glycol diethyl ether to $200{ }^{\circ} \mathrm{C}$ where the very weak fluorescence reversibly becomes intensified by a factor of 2 (Fig. S36, ESI $\dagger$ ). ${ }^{17}$ This is attributed to thermally induced vibronic perturbation of the nearly orthogonal arrangement enabling fluorescence. The proposed geometrical requirements for a distinct charge transfer are related to the TICT theory. ${ }^{18}$ However, our results imply that orthogonal arrangements between the donor and the acceptor completely quench the fluorescence. Significant fluorescence is attributed to skew conformations which tend to more planar arrangements in the excited state allowing significant orbital overlap. Thus, the optical properties, particularly the fluorescent solvatochromism is not the result of a twist-induced charge transfer. It is rather the result of an interplay of conformational change and electronic charge transfer depending on the dipole moment. By tuning the molecular geometry we could obtain a series of chromophores with adjustable fluorescence and quantum yields of up to more than $80 \%$.

\section{Conclusions}

In summary, we have reported new, readily soluble and highly fluorescent derivatives of naphthalene-1,8-dicarboximides that have been obtained by Pd-catalyzed arylation in position 4 . These compounds display a pronounced solvatochromic fluorescence. The sensitivity of the substituted naphthalimides towards solvent polarity was evaluated according to several theoretical approaches. A photo-induced charge transfer from the electron rich aryl moiety to the naphthalimide is enhanced in polar solvents and causes a bathochromic shift of the fluorescence. Furthermore, the electronic effects are accompanied by molecular dynamics. The intramolecular arrangement influences the intensity of the charge transfer. A skew geometry between the donor and the acceptor allows planarization in the first electronically excited state. This allows high fluorescent quantum yields and still favours a pronounced charge transfer resulting in both distinct solvatochromism and large Stokes shifts. In contrast to basic TICTtheory, no orthogonalization occurs and rectangular orientation leads to strongly quenched fluorescence. The presented synergy of electronic and geometric effects results in highly fluorescent compounds such as $\mathbf{3 b}$ and $\mathbf{3 g}$ with easily adjustable emission spectra controlled by medium effects. This provides very large Stokes shifts exceeding $200 \mathrm{~nm}$ (approx. $1.6 \mathrm{eV}$ ) being of interest for various applications such as for frequency converters, fluorescence optical fibers and highly tunable light sources.

\section{Experimental}

General information on the experimental conduct and synthesis of precursors can be found in the ESI. $\dagger$

\section{6-Phenyl-2-(tridecan-7-yl)-1H-benzo[de]isoquinoline-1,3(2H)- dione (3a)}

A Schlenk-flask was charged with 6-bromo-2-(tridecan-7-yl)$1 H$-benzo[de]isoquinoline-1,3(2H)-dione (480 mg, $1.05 \mathrm{mmol}$, 1.0 equiv.) and 4,4,5,5-tetramethyl-2-phenyl-1,3,2-dioxaborolane (265 mg, $1.30 \mathrm{mmol}, 1.2$ equiv.). Both compounds were dissolved in toluene $(20 \mathrm{~mL})$ under a light argon-stream. $\mathrm{K}_{2} \mathrm{CO}_{3}(2.76 \mathrm{~g}$, $20.0 \mathrm{mmol})$ was dissolved in a mixture of water $(10 \mathrm{~mL})$ and EtOH $(4 \mathrm{~mL})$ and added to the Schlenk-flask followed by tetrakis(triphenylphosphine)palladium(0) (116 mg, $0.100 \mathrm{mmol}$, $10 \mathrm{~mol} \%$ ). The mixture was purged with argon for $30 \mathrm{~min}$ and then heated to $80{ }^{\circ} \mathrm{C}$. The reaction mixture was stirred at this temperature for further $17 \mathrm{~h}$. After cooling to room temperature, the organic phase was separated and the aqueous phase was extracted with toluene $(3 \times 10 \mathrm{~mL})$. Purification by column chromatography (silica, iso-hexane $/ \mathrm{CHCl}_{3}=1: 1$ ) gave compound 3a as a bright yellow solid (450 mg, 94\%). M.p.: $97{ }^{\circ} \mathrm{C}$. IR (diamond-ATR, neat): $\tilde{\nu} / \mathrm{cm}^{-1}=2919,2852,1696,1653,1587$, 1396, 1348, 1237, 1176, 1100, 784, 768, 702. ${ }^{1} \mathrm{H}$ NMR $\left(\mathrm{CDCl}_{3}\right.$, $600 \mathrm{MHz}): \delta / \mathrm{ppm}=8.69-8.56(\mathrm{~m}, 2 \mathrm{H}), 8.25(\mathrm{dd}, J=8.5,1.1 \mathrm{~Hz}$, $1 \mathrm{H}), 7.74-7.66(\mathrm{~m}, 1 \mathrm{H}), 7.58-7.53(\mathrm{~m}, 2 \mathrm{H}), 7.53-7.48(\mathrm{~m}, 3 \mathrm{H})$, 5.23-5.16 (m, 1H, NCH), 2.31-2.20 (m, $\left.2 \mathrm{H}, \beta-\mathrm{CH}_{2}\right), 1.89-1.80$ $\left(\mathrm{m}, 2 \mathrm{H}, \beta-\mathrm{CH}_{2}\right), 1.40-1.16\left(\mathrm{~m}, 16 \mathrm{H}, 8 \times \mathrm{CH}_{2}\right), 0.83(\mathrm{t}, J=7.1 \mathrm{~Hz}$, $6 \mathrm{H}) .{ }^{13} \mathrm{C} \mathrm{NMR}\left(\mathrm{CDCl}_{3}, 150 \mathrm{MHz}\right): \delta / \mathrm{ppm}=165.68,165.48$, 164.60, 164.41, 146.64, 139.04, 132.40, 131.70, 131.34, 130.91, 130.56, 130.08, 129.99, 129.00, 128.79, 128.56, 127.97, 126.97, 123.72, 123.00, 122.61, 121.88, 54.63, 32.58, 31.92, 29.39, 27.06,

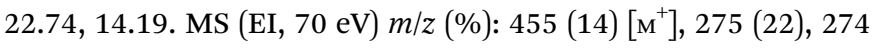
(100), 273 (22), 256 (12), 202 (8). HRMS (EI) for $\mathrm{C}_{31} \mathrm{H}_{37} \mathrm{NO}_{2}$ : calcd: 455.2824; found: 455.2820. EA for $\mathrm{C}_{31} \mathrm{H}_{37} \mathrm{NO}_{2}$ : calcd: C 81.72, N 3.07, H 8.19; found: C 81.52, N 3.10, H 8.31. UV/Vis $\left(\mathrm{CHCl}_{3}\right): \lambda_{\max }(\varepsilon)=355.4 \mathrm{~nm}(17100)$. Fluorescence $\left(\mathrm{CHCl}_{3}\right.$, $\left.\lambda_{\text {exc }}=355.4 \mathrm{~nm}\right): \lambda_{\max }=420.0 \mathrm{~nm}$. Fluorescence quantum yield 
$\left(\mathrm{CHCl}_{3}, \lambda_{\text {exc }}=355.4 \mathrm{~nm}, E_{355.4 \mathrm{~nm}, 1 \mathrm{~cm}}=0.134\right.$, reference: $N, N^{\prime}$-bis(tridecan-7-yl)perylene-3,4:9,10-tetracarboxylic diimide with $\Phi=1.00): \Phi=0.78$.

\section{6-(4-Methoxyphenyl)-2-(tridecan-7-yl)-1H- benzo[de]isoquinoline-1,3(2H)-dione $(3 \mathrm{~b})$}

A Schlenk-flask was charged with 6-bromo-2-(tridecan-7-yl)$1 H$-benzo[de]isoquinoline-1,3(2H)-dione $(215 \mathrm{mg}, 0.470 \mathrm{mmol}$, 1.04 equiv.) and 2-(4-methoxyphenyl)-4,4,5,5-tetramethyl-1,3,2dioxaborolane (100 mg, $0.450 \mathrm{mmol}, 1.0$ equiv.). Both compounds were dissolved in toluene $(10 \mathrm{~mL})$ under a light argon-stream. $\mathrm{K}_{2} \mathrm{CO}_{3}(1.40 \mathrm{~g}, 10.0 \mathrm{mmol})$ was dissolved in a mixture of water $(5 \mathrm{~mL})$ and EtOH $(1.00 \mathrm{~mL})$ and added to the Schlenk-flask followed by tetrakis(triphenylphosphine)palladium(0) $(20 \mathrm{mg}$, $0.018 \mathrm{mmol}, 5 \mathrm{~mol} \%)$. The mixture was purged with argon for $30 \mathrm{~min}$ and then heated to $80{ }^{\circ} \mathrm{C}$. The reaction mixture was stirred at this temperature for further $17 \mathrm{~h}$. After cooling to room temperature, the organic phase was separated and the aqueous phase was extracted with toluene $(3 \times 10 \mathrm{~mL})$. Purification by column chromatography (silica, iso-hexane $/ \mathrm{CHCl}_{3}=1: 1$ ) gave compound $3 \mathbf{b}$ as an off-white solid (176 mg, 78\%). M.p.: $63{ }^{\circ} \mathrm{C}$. IR (diamond-ATR, neat): $\tilde{\nu} / \mathrm{cm}^{-1}=2922,2852,1698,1656,1608$, 1588, 1518, 1506, 1463, 1397, 1348, 1288, 1239, 1176, 1096, 1078, 1036, 963, 867, 838, 785, 760, 724. ${ }^{1} \mathrm{H} \mathrm{NMR}\left(\mathrm{CDCl}_{3}, 600 \mathrm{MHz}\right)$ : $\delta / \mathrm{ppm}=8.66-8.52(\mathrm{br} \mathrm{s}, 2 \mathrm{H}), 8.29(\mathrm{dd}, J=8.5 \mathrm{~Hz}, 1.1 \mathrm{~Hz}, 1 \mathrm{H})$, $7.72-7.63(\mathrm{~m}, 2 \mathrm{H}), 7.44(\mathrm{~d}, J=8.8 \mathrm{~Hz}, 2 \mathrm{H}), 7.07(\mathrm{~d}, J=8.8 \mathrm{~Hz}$, $2 \mathrm{H}), 5.26-5.13(\mathrm{~m}, 1 \mathrm{H}, \mathrm{NCH}), 3.90\left(\mathrm{~s}, 3 \mathrm{H}, \mathrm{OCH}_{3}\right), 2.33-2.17(\mathrm{~m}$, $\left.2 \mathrm{H}, \beta-\mathrm{CH}_{2}\right), 1.91-1.77\left(\mathrm{~m}, 2 \mathrm{H}, \beta-\mathrm{CH}_{2}\right), 1.42-1.11(\mathrm{~m}, 16 \mathrm{H}$, $\left.8 \times \mathrm{CH}_{2}\right), 0.82(\mathrm{t}, J=9.0 \mathrm{~Hz}, 6 \mathrm{H}) .{ }^{13} \mathrm{C} \mathrm{NMR}\left(\mathrm{CDCl}_{3}, 150 \mathrm{MHz}\right)$ : $\delta / \mathrm{ppm}=165.54,164.54,160.02,146.43,132.45,131.30,131.20$, $130.17,129.07,127.86,126.80,123.70,123.06,122.18,121.55$, $114.28,55.52,54.55,32.56,31.89,29.36,27.03,22.70,14.15 . \mathrm{MS}$

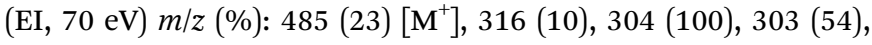
286 (13), 198 (34), 180 (12). HRMS (EI) for $\mathrm{C}_{32} \mathrm{H}_{39} \mathrm{NO}_{3}$ : calcd: 485.2930; found: 485.2924. EA for $\mathrm{C}_{32} \mathrm{H}_{39} \mathrm{NO}_{3}$ : calcd: $\mathrm{C} 79.14, \mathrm{~N}$ 2.88, H 8.09; found: C 79.18, N 2.69, $\mathrm{H}$ 8.22. UV/Vis $\left(\mathrm{CHCl}_{3}\right)$ : $\lambda_{\text {max }}(\varepsilon)=364.8 \mathrm{~nm}(16900)$. Fluorescence $\left(\mathrm{CHCl}_{3}, \lambda_{\text {exc }}=364.8 \mathrm{~nm}\right)$ : $\lambda_{\text {max }}=459.6 \mathrm{~nm}$. Fluorescence quantum yield $\left(\mathrm{CHCl}_{3}, \lambda_{\text {exc }}=\right.$ $364.8 \mathrm{~nm}, E_{364.8 \mathrm{~nm}, 1 \mathrm{~cm}}=0.252$, reference: $N, N^{\prime}$-bis(tridecan-7yl)perylene-3,4:9,10-tetracarboxylic diimide with $\Phi=1.00$ ): $\Phi=0.83$.

\section{6-(4-(Dimethylamino)phenyl)-2-(tridecan-7-yl)-1H-} benzo[de]isoquinoline-1,3(2H)-dione (3c)

A Schlenk-flask was charged with 6-bromo-2-(tridecan-7-yl)$1 H$-benzo[de]isoquinoline-1,3(2H)-dione $(480 \mathrm{mg}, 1.05 \mathrm{mmol}$, 1.05 equiv.) and $N, N$-dimethyl-4-(4,4,5,5-tetramethyl-1,3,2-dioxaborolan-2-yl)aniline ( $247 \mathrm{mg}, 1.00 \mathrm{mmol}, 1.0$ equiv.). Both compounds were dissolved in toluene $(20 \mathrm{~mL})$ under a light argon stream. $\mathrm{K}_{2} \mathrm{CO}_{3}(2.80 \mathrm{~g}, 20.0 \mathrm{mmol})$ was dissolved in a mixture of water $(10 \mathrm{~mL})$ and $\mathrm{EtOH}(3 \mathrm{~mL})$ and added to the Schlenk-flask followed by tetrakis(triphenylphosphine)palladium(0) $(115 \mathrm{mg}$, $0.100 \mathrm{mmol}, 10 \mathrm{~mol} \%)$. The mixture was purged with argon for $30 \mathrm{~min}$ and then heated to $80{ }^{\circ} \mathrm{C}$. The reaction mixture was stirred at this temperature for further $17 \mathrm{~h}$. After cooling to room temperature, the organic phase was separated and the aqueous phase was extracted with toluene $(3 \times 10 \mathrm{~mL})$. Purification by column chromatography (silica, iso-hexane $/ \mathrm{CHCl}_{3}=5: 2$ ) gave compound 3c as a bright yellow solid (70 mg, 14\%). M.p.: $125{ }^{\circ} \mathrm{C}$. IR (diamond-ATR, neat): $\tilde{\nu} / \mathrm{cm}^{-1}=2921,2852,1694,1654,1609$, 1587, 1524, 1465, 1397, 1349, 1237, 1199, 1101, 943, 872, 818, $785,761 .{ }^{1} \mathrm{H} \mathrm{NMR}\left(\mathrm{CDCl}_{3}, 600 \mathrm{MHz}\right): \delta / \mathrm{ppm}=8.64-8.54(\mathrm{~m}, 2 \mathrm{H})$, $8.40(\mathrm{~d}, J=8.5 \mathrm{~Hz}, 1 \mathrm{H}), 7.70-7.65(\mathrm{~m}, 2 \mathrm{H}), 7.43(\mathrm{~d}, J=8.7 \mathrm{~Hz}, 2 \mathrm{H})$, $6.88(\mathrm{~d}, J=8.7 \mathrm{~Hz}, 2 \mathrm{H}), 5.22-5.15(\mathrm{~m}, 1 \mathrm{H}), 3.07\left(\mathrm{~s}, 6 \mathrm{H}, \mathrm{NMe}_{2}\right)$, 2.29-2.20 (m, 2H, $\beta-\mathrm{CH}_{2}$ ), 1.88-1.80 (m, 2H, $\left.\beta-\mathrm{CH}_{2}\right), 1.35-1.18$ $\left(\mathrm{m}, 16 \mathrm{H}, 8 \times \mathrm{CH}_{2}\right), 0.82(\mathrm{t}, J=7.0 \mathrm{~Hz}, 6 \mathrm{H}) .{ }^{13} \mathrm{C} \mathrm{NMR}\left(\mathrm{CDCl}_{3}\right.$, $150 \mathrm{MHz}): \delta / \mathrm{ppm}=165.87,165.64,164.79,164.61,150.65$, $147.29,132.92$, 131.57, 131.08, 130.77, 130.17, 129.28, 127.57, 126.59, 126.53, 112.16, 123.66, 122.95, 121.47, 120.75, 54.53, $40.57,32.62,31.93,29.41,27.07,22.57,14.20$. MS (EI, $70 \mathrm{eV}$ ): $\mathrm{m} / z(\%)=499$ (22), 498 (59) [ $\left.\mathrm{M}^{+}\right], 318$ (10), 317 (43), 316 (100), 315 (19). HRMS (EI) for $\mathrm{C}_{33} \mathrm{H}_{42} \mathrm{~N}_{2} \mathrm{O}_{2}$ : calcd: 498.3246; found: 498.3227. EA for $\mathrm{C}_{33} \mathrm{H}_{42} \mathrm{~N}_{2} \mathrm{O}_{2}$ : calcd: $\mathrm{C}$ 79.48, N 5.62, H 8.49; found: C 79.42, N 5.64, $\mathrm{H} 8.36$. UV/Vis $\left(\mathrm{CHCl}_{3}\right): \lambda_{\max }(\varepsilon)=426.2 \mathrm{~nm}$ (17100). Fluorescence $\left(\mathrm{CHCl}_{3}, \lambda_{\text {exc }}=426.2 \mathrm{~nm}\right): \lambda_{\max }=$ $578.4 \mathrm{~nm}$. Fluorescence quantum yield $\left(\mathrm{CHCl}_{3}, \lambda_{\text {exc }}=426.2 \mathrm{~nm}\right.$, $E_{426.2 \mathrm{~nm}, 1 \mathrm{~cm}}=0.114$, reference: $N, N^{\prime}$-bis(tridecan-7-yl)perylene3,4:9,10-tetracarboxylic diimide with $\Phi=1.00): ~ \Phi=0.64$.

\section{6-(3,4,5-Trimethoxyphenyl)-2-(tridecan-7-yl)-1H- benzo[de]isoquinoline-1,3(2H)-dione (3d)}

A Schlenk-flask was charged with 6-bromo-2-(tridecan-7-yl)$1 H$-benzo[de]isoquinoline-1,3(2H)-dione (361 mg, $0.788 \mathrm{mmol}$, 1.05 equiv.) and 4,4,5,5-tetramethyl-2-(3,4,5-trimethoxyphenyl)1,3,2-dioxaborolane (220 mg, $0.750 \mathrm{mmol}, 1.0$ equiv.). Both compounds were dissolved in toluene $(20 \mathrm{~mL})$ under a light argon-stream. $\mathrm{K}_{2} \mathrm{CO}_{3}(2.80 \mathrm{~g}, 20.0 \mathrm{mmol})$ was dissolved in a mixture of water $(10 \mathrm{~mL})$ and $\mathrm{EtOH}(2 \mathrm{~mL})$ and added to the Schlenk-flask followed by tetrakis(triphenylphosphine)palladium(0) (43 mg, $0.038 \mathrm{mmol}, 5 \mathrm{~mol} \%$ ). The mixture was purged with argon for $30 \mathrm{~min}$ and then heated to $80{ }^{\circ} \mathrm{C}$. The reaction mixture was stirred at this temperature for further $17 \mathrm{~h}$. After cooling to room temperature, the organic phase was separated and the aqueous phase was extracted with toluene $(3 \times 20 \mathrm{~mL})$. Purification by column chromatography ( silica, $\mathrm{CHCl}_{3}$ ) gave compound $\mathbf{3 d}$ as yellow oil (350 mg, 86\%). IR (diamond-ATR, neat): $\tilde{\nu} / \mathrm{cm}^{-1}=$ 2924, 2855, 1698, 1656, 1616, 1586, 1503, 1454, 1415, 1397, 1350, 1322, 1237, 1180, 1126, 1104, 1060, 1006, 928, 912, 860, 842, 831, 785, 760, 725, 702, 678. ${ }^{1} \mathrm{H} \mathrm{NMR}\left(\mathrm{CDCl}_{3}, 400 \mathrm{MHz}\right): \delta / \mathrm{ppm}=8.69$ (br s, 2H), 8.31 (dd, $J=8.5,1.1 \mathrm{~Hz}, 1 \mathrm{H}), 7.71(\mathrm{t}, J=7.1 \mathrm{~Hz}, 1 \mathrm{H})$, $6.70(\mathrm{~s}, 2 \mathrm{H}), 5.24-5.13(\mathrm{~m}, 1 \mathrm{H}, \mathrm{NCH}), 3.95$ (s, 3H, OMe), 3.89 (s, $6 \mathrm{H}, \mathrm{OMe}), 2.31-2.18\left(\mathrm{~m}, 2 \mathrm{H}, \beta-\mathrm{CH}_{2}\right), 1.90-1.77\left(\mathrm{~m}, 2 \mathrm{H}, \beta-\mathrm{CH}_{2}\right)$, 1.35-1.16 (m, 16H, $\left.8 \times \mathrm{CH}_{2}\right), 0.81(\mathrm{t}, J=6.9 \mathrm{~Hz}, 6 \mathrm{H}) .{ }^{13} \mathrm{C} \mathrm{NMR}$ $\left(\mathrm{CDCl}_{3}, 75 \mathrm{MHz}\right): \delta / \mathrm{ppm}=165.56,165.36,164.50,164.31,153.45$, $146.60,138.39,134.53,132.35,131.71,131.23,130.94,130.42$, $130.14,128.94,127.71,126.99,123.66,122.97,122.55,121.84$, 107.30, 61.12, 56.42, 54.58, 32.52, 31.87, 29.33, 27.00, 22.67, 14.14. MS (EI, $70 \mathrm{eV}): m / z(\%)=545(40)\left[\mathrm{M}^{+}\right], 365$ (16), 364 (76), 363 (100), 348 (19). HRMS (EI) for $\mathrm{C}_{34} \mathrm{H}_{43} \mathrm{NO}_{5}$ : calcd: 545.3141; found: 545.3135. EA for $\mathrm{C}_{34} \mathrm{H}_{43} \mathrm{NO}_{5}$ : calcd: $\mathrm{C} 74.83$, $\mathrm{N} 2.57, \mathrm{H}$ 7.94; found: $\mathrm{C} 73.47, \mathrm{~N} 2.58, \mathrm{H}$ 7.75. UV/Vis $\left(\mathrm{CHCl}_{3}\right)$ : $\lambda_{\text {max }}(\varepsilon)=362.2 \mathrm{~nm}$ (15900). Fluorescence $\left(\mathrm{CHCl}_{3}, \lambda_{\text {exc }}=\right.$ $362.2 \mathrm{~nm}): \lambda_{\max }=525.1 \mathrm{~nm}$. Fluorescence quantum yield 
$\left(\mathrm{CHCl}_{3}, \lambda_{\text {exc }}=362.2 \mathrm{~nm}, E_{362.2 \mathrm{~nm}, 1 \mathrm{~cm}}=0.221\right.$, reference: $N, N^{\prime}$-bis(tridecan-7-yl)perylene-3,4:9,10-tetracarboxylic diimide with $\Phi=1.00)$ : $\Phi=0.65$.

\section{6-(2,6-Dibromo-3,4,5-trimethoxyphenyl)-2-(tridecan-7-yl)-1H- benzo[de]isoquinoline-1,3(2H)-dione (3e)}

6-(3,4,5-Trimethoxyphenyl)-2-(tridecan-7-yl)-1H-benzo[de]iso-quinoline-1,3(2H)-dione (175 mg, $0.320 \mathrm{mmol}$ ) was dissolved in $10 \mathrm{~mL}$ of acetonitrile. $N$-Bromosuccinimide $(120 \mathrm{mg}, 0.670 \mathrm{mmol}, 2.1$ equiv.) was added at once to the solution and the reaction mixture was stirred at $30{ }^{\circ} \mathrm{C}$ for $12 \mathrm{~h}$. The crude mixture was treated with brine solution and extracted with chloroform. The organic phase was dried over $\mathrm{MgSO}_{4}$ and concentrated in vacuo. Column chromatography $\left(\mathrm{CHCl}_{3}\right)$ gave $3 \mathrm{e}$ as colorless oil (155 mg, 69\%). IR (diamond-ATR, neat): $\tilde{\nu} / \mathrm{cm}^{-1}=2925,2855,1699,1658,1590$, 1461, 1397, 1352, 1321, 1237, 1179, 1087, 1008, 986, 935, 909, 861, 784, 763, 729. ${ }^{1} \mathrm{H}$ NMR $\left(\mathrm{CDCl}_{3}, 600 \mathrm{MHz}\right): \delta / \mathrm{ppm}=8.68-8.54$ (m, 2H), 7.72-7.65 (m, 2H), 7.56 (d, $J=7.5 \mathrm{~Hz}, 1 \mathrm{H}), 5.22-5.15(\mathrm{~m}$, $1 \mathrm{H}, \mathrm{NCH}), 4.06$ (s, 3H, OMe), 3.98 (s, 6H, OMe), 2.28-2.20 (m, $2 \mathrm{H}$, $\left.\beta-\mathrm{CH}_{2}\right), 1.88-1.82\left(\mathrm{~m}, 2 \mathrm{H}, \beta-\mathrm{CH}_{2}\right), 1.36-1.20\left(\mathrm{~m}, 16 \mathrm{H}, 8 \times \mathrm{CH}_{2}\right)$, $0.82(\mathrm{t}, J=7.0 \mathrm{~Hz}, 6 \mathrm{H}) \cdot{ }^{13} \mathrm{C} \mathrm{NMR}\left(\mathrm{CDCl}_{3}, 150 \mathrm{MHz}\right): \delta / \mathrm{ppm}=$ $165.43,165.21,164.36,164.14,151.23,147.87,144.79,135.49$, $131.82,131.28,131.06,130.51,129.57,128.63,128.56,127.41$, $123.93,123.93,123.51,123.20,122.78,114.58,61.54,61.28$, 54.64, 32.56, 31.86, 29.37, 27.05, 22.73, 14.16. MS (EI, $70 \mathrm{eV})$

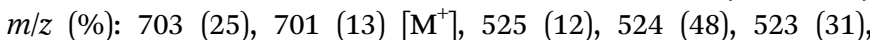
522 (100), 521 (31), 520 (50), 399 (9), 397 (9), 361 (25). HRMS (EI) for $\mathrm{C}_{34} \mathrm{H}_{41} \mathrm{Br}_{2} \mathrm{NO}_{5}$ : calcd: 701.1351; found: 701.1357.

\section{6-(3,4,5-Trimethoxy-2,6-dimethylphenyl)-2-(tridecan-7-yl)- $1 H$ - benzo[de]isoquinoline-1,3(2H)-dione (3f)}

A dry argon flushed Schlenk-flask was charged with $\mathrm{ZnCl}_{2}$ solution (0.43 mL, $0.43 \mathrm{mmol}, 1 \mathrm{M}, 2.1$ equiv.). $\mathrm{MeMgCl}(0.14 \mathrm{~mL}$, $0.43 \mathrm{mmol}, 3.0 \mathrm{M}, 2.1$ equiv.) was added dropwise to the solution at $0{ }^{\circ} \mathrm{C}$. Another dry argon flushed Schlenk-flask was charged with 6-(2,6-dibromo-3,4,5-trimethoxyphenyl)-2-(tridecan7-yl)-1H-benzo[de]isoquinoline-1,3(2H)-dione (145 mg, $0.200 \mathrm{mmol}$, 1.0 equiv.), $\mathrm{Pd}(\mathrm{OAc})_{2}(3.0 \mathrm{mg}, 0.010 \mathrm{mmol}, 5 \mathrm{~mol} \%)$ and $S$-Phos ( $9.0 \mathrm{mg}, 0.020 \mathrm{mmol}, 10 \mathrm{~mol} \%$ ) in $1.0 \mathrm{~mL}$ of dry THF. After stirring for $10 \mathrm{~min}$, this solution was added dropwise to the freshly prepared $\mathrm{MeZnCl}$ solution. The reaction mixture was stirred at $60{ }^{\circ} \mathrm{C}$ over night and then quenched with sat. aq. $\mathrm{NH}_{4} \mathrm{Cl}$ solution. The crude mixture was extracted with chloroform and concentrated in vacuo. Column chromatography (silica, $\mathrm{CHCl}_{3}$ ) gave $3 \mathbf{f}$ as colorless oil (85.0 mg, 74\%). IR (diamond-ATR, neat): $\tilde{\nu} / \mathrm{cm}^{-1}=2925,2856,1700,1658,1589,1456,1399,1349,1320$, 1237, 1110, 1081, 862, 785, 764. ${ }^{1} \mathrm{H}$ NMR $\left(\mathrm{CDCl}_{3}, 300 \mathrm{MHz}\right)$ : $\delta / \mathrm{ppm}=8.67-8.52($ br s, $2 \mathrm{H}), 7.71(\mathrm{dd}, J=8.4,1.2 \mathrm{~Hz}, 1 \mathrm{H})$, $7.65-7.59(\mathrm{~m}, 1 \mathrm{H}), 7.51(\mathrm{~d}, J=7.4 \mathrm{~Hz}, 1 \mathrm{H}), 5.25-5.13(\mathrm{~m}, 1 \mathrm{H}$, $\mathrm{NCH}), 4.02$ (s, 3H, OMe), 3.89 (s, 6H, OMe), 2.30-2.20 (m, $2 \mathrm{H}$, $\left.\beta-\mathrm{CH}_{2}\right), 1.90-1.80\left(\mathrm{~m}, 2 \mathrm{H}, \beta-\mathrm{CH}_{2}\right), 1.75\left(\mathrm{~s}, 6 \mathrm{H}, 2 \times \mathrm{CH}_{3}\right)$, 1.35-1.19 (m, 16H, $\left.8 \times \mathrm{CH}_{2}\right), 0.81(\mathrm{t}, J=6.9 \mathrm{~Hz}, 6 \mathrm{H})$. ${ }^{13} \mathrm{C} \mathrm{NMR}\left(\mathrm{CDCl}_{3}, 150 \mathrm{MHz}\right): \delta / \mathrm{ppm}=165.58,164.47,150.13$, 146.23, 145.44, 133.80, 131.68, 131.56, 130.97, 130.79, 130.50, 128.81, 128.22, 127.19, 125.85, 123.88, 123.14, 122.64, 121.90, $60.93,60.86,54.59,32.58,31.89,29.39,27.05,22.74,14.16,13.50$.

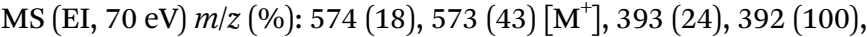
391 (92), 348 (7), 55 (8). HRMS (EI) for $\mathrm{C}_{36} \mathrm{H}_{47} \mathrm{NO}_{5}$ : calcd: 573.3454; found: 573.3448. UV/Vis $\left(\mathrm{CHCl}_{3}\right): \lambda_{\max }(\varepsilon)=358.2$ (14300), $344.0 \mathrm{~nm}$ (16 000). Fluorescence $\left(\mathrm{CHCl}_{3}, \lambda_{\text {exc }}=344.0 \mathrm{~nm}\right)$ : $\lambda_{\text {max }}=541.6 \mathrm{~nm}$. Fluorescence quantum yield $\left(\mathrm{CHCl}_{3}, \lambda_{\text {exc }}=\right.$ $344.0 \mathrm{~nm}, E_{344.0 \mathrm{~nm}, 1 \mathrm{~cm}}=0.094$, reference: $N, N^{\prime}$-bis(tridecan7-yl)perylene-3,4:9,10-tetracarboxylic diimide with $\Phi=1.00)$ : $\Phi=0.046$.

\section{6-(4-Methoxynaphthalen-1-yl)-2-(tridecan-7-yl)-1H- benzo[de]isoquinoline-1,3(2H)-dione $(3 \mathrm{~g})$}

A Schlenk-flask was charged with 6-bromo-2-(tridecan-7-yl)$1 H$-benzo[de]isoquinoline-1,3(2H)-dione (348 mg, $0.760 \mathrm{mmol}$, 1.08 equiv.) and 2-(4-methoxynaphthalen-1-yl)-4,4,5,5-tetramethyl1,3,2-dioxaborolane (200 mg, $0.700 \mathrm{mmol}, 1.0$ equiv.). Both compounds were dissolved in toluene $(10 \mathrm{~mL})$ under a light argon-stream. $\mathrm{K}_{2} \mathrm{CO}_{3}(1.40 \mathrm{~g}, 10.0 \mathrm{mmol})$ was dissolved in a mixture of water $(5 \mathrm{~mL})$ and $\mathrm{EtOH}(1.0 \mathrm{~mL})$ and added to the Schlenk-flask followed by tetrakis-(triphenylphosphine)palladium(0) (20 mg, $0.018 \mathrm{mmol}, 5 \mathrm{~mol} \%$ ). The mixture was purged with argon for $30 \mathrm{~min}$ and then heated to $80{ }^{\circ} \mathrm{C}$. The reaction mixture was stirred at this temperature for further $17 \mathrm{~h}$. After cooling to room temperature, the organic phase was separated and the aqueous phase was extracted with toluene $(3 \times 20 \mathrm{~mL})$. Purification by column chromatography (silica, iso-hexane $/ \mathrm{CHCl}_{3}=1: 1$ ) gave compound $3 \mathrm{~g}$ as a bright yellow solid (260 $\mathrm{mg}, 69 \%)$. M.p.: $98{ }^{\circ} \mathrm{C}$. IR (diamond-ATR, neat): $\tilde{\nu} / \mathrm{cm}^{-1}=2920,2853,1697,1655$, 1615, 1586, 1511, 1459, 1421, 1398, 1348, 1311, 1235, 1177, 1157, 1106, 1084, 1025, 881, 817, 784, 759, 735, 712, 667. ${ }^{1} \mathrm{H}$ NMR $\left(\mathrm{CDCl}_{3}, 300 \mathrm{MHz}\right): \delta / \mathrm{ppm}=8.74-8.64(\mathrm{br} \mathrm{s}, 1 \mathrm{H}), 8.64-8.52(\mathrm{br} \mathrm{s}$, $1 \mathrm{H}), 8.41(\mathrm{~d}, J=8.5 \mathrm{~Hz}, 1 \mathrm{H}), 7.82(\mathrm{~d}, J=8.4 \mathrm{~Hz}, 1 \mathrm{H}), 7.76(\mathrm{~d}, J=$ $7.5 \mathrm{~Hz}, 1 \mathrm{H}), 7.57-7.46$ (m, 2H), 7.39 (d, $J=7.9 \mathrm{~Hz}, 1 \mathrm{H}), 7.36-7.27$ $(\mathrm{m}, 2 \mathrm{H}), 6.97(\mathrm{~d}, J=7.9 \mathrm{~Hz}, 1 \mathrm{H}) 5.32-5.17(\mathrm{~m}, 1 \mathrm{H}, \mathrm{NCH}), 4.11(\mathrm{~s}$, $3 \mathrm{H}, \mathrm{OMe}$ ), 2.39-2.22 (m, 2H, $\left.\beta-\mathrm{CH}_{2}\right), 1.96-1.81$ (m, 2H, $\left.\beta-\mathrm{CH}_{2}\right)$, 1.47-1.15 (m, 16H, $\left.8 \times \mathrm{CH}_{2}\right), 0.85(\mathrm{t}, J=6.6 \mathrm{~Hz}, 6 \mathrm{H}) .{ }^{13} \mathrm{C} \mathrm{NMR}$ $\left(\mathrm{CDCl}_{3}, 75 \mathrm{MHz}\right): \delta / \mathrm{ppm}=165.51,164.62,156.09,145.53$, 133.30, 132.85, 131.64, 131.24, 130.92, 130.50, 129.36, 128.74, 128.69, 128.13, 127.13, 126.82, 125.79, 125.68, 125.61, 122.52, 103.39, 55.80, 54.60, 32.61, 31.92, 29.40, 27.07, 22.74, 14.18. MS (EI, $70 \mathrm{eV}) \mathrm{m} / z$ (\%): 535 (32) [ $\left.\mathrm{M}^{+}\right], 355$ (16), 354 (69), 353 (100), 239 (8). HRMS (EI) for $\mathrm{C}_{36} \mathrm{H}_{41} \mathrm{NO}_{3}$ : calcd: 535.3086; found: 535.3080. EA for $\mathrm{C}_{36} \mathrm{H}_{41} \mathrm{NO}_{3}$ : calcd: $\mathrm{C}$ 80.71, N 2.61, H 7.71; found: $\mathrm{C}$ 80.86, $\mathrm{N} 2.49, \mathrm{H}$ 7.84. UV/Vis $\left(\mathrm{CHCl}_{3}\right): \lambda_{\max }(\varepsilon)=$ $325.0 \mathrm{~nm}$ (15300). Fluorescence $\left(\mathrm{CHCl}_{3}, \lambda_{\text {exc }}=325.0 \mathrm{~nm}\right)$ : $\lambda_{\text {max }}=509.7 \mathrm{~nm}$. Fluorescence quantum yield $\left(\mathrm{CHCl}_{3}, \lambda_{\text {exc }}=\right.$ $325.0 \mathrm{~nm}, E_{325.0 \mathrm{~nm}, 1 \mathrm{~cm}}=0.139$, reference: $N, N^{\prime}$-bis(tridecan7-yl)perylene-3,4:9,10-tetracarboxylic diimide with $\Phi=1.00)$ : $\Phi=0.39$.

\section{6-(4-Methoxy-8-methylnaphthalen-1-yl)-2-(tridecan-7-yl)-1H- benzo[de]isoquinoline-1,3(2H)-dione $(3 \mathrm{~h})$}

A Schlenk-flask was charged with 6-bromo-2-(tridecan-7-yl)$1 H$-benzo[de]isoquinoline-1,3(2H)-dione (124 mg, $0.273 \mathrm{mmol}$, 1.05 equiv.) and 2-(4-methoxy-8-methylnaphthalen-1-yl)-4,4,5,5tetramethyl-1,3,2-dioxaborolane ( $77 \mathrm{mg}, 0.26 \mathrm{mmol}, 1.0$ equiv.). Both compounds were dissolved in toluene $(5 \mathrm{~mL})$ under a 
light argon-stream. $\mathrm{K}_{2} \mathrm{CO}_{3}(0.700 \mathrm{~g}, 5.07 \mathrm{mmol})$ was dissolved in a mixture of water $(3 \mathrm{~mL})$ and $\mathrm{EtOH}(0.5 \mathrm{~mL})$ and added to the Schlenk-flask followed by tetrakis-(triphenylphosphine)palladium(0) (15 mg, $0.013 \mathrm{mmol}, 5 \mathrm{~mol} \%$ ). The mixture was purged with argon for $30 \mathrm{~min}$ and then heated to $80{ }^{\circ} \mathrm{C}$. The reaction mixture was stirred at this temperature for further $17 \mathrm{~h}$. After cooling to room temperature, the organic phase was separated and the aqueous phase was extracted with toluene $(3 \times 5 \mathrm{~mL})$. Purification by column chromatography (silica, iso-hexane $/ \mathrm{CHCl}_{3}=1: 1$ ) gave compound $3 \mathbf{h}$ as a bright yellow solid (36.0 $\mathrm{mg}, 25 \%)$. M.p.: $164{ }^{\circ} \mathrm{C}$. IR (diamond-ATR, neat): $\tilde{\nu} / \mathrm{cm}^{-1}=2920,2853,1697$, 1656, 1587, 1513, 1450, 1397, 1344, 1314, 1233, 1153, 1099, 1045, 815, 784, 765, 726, 677. ${ }^{1} \mathrm{H} \mathrm{NMR}\left(\mathrm{CDCl}_{3}, 600 \mathrm{MHz}\right)$ : $\delta / \mathrm{ppm}=8.66-8.50(\mathrm{br}, 2 \mathrm{H}), 8.38(\mathrm{~d}, J=7.9 \mathrm{~Hz}, 1 \mathrm{H}), 7.76(\mathrm{dd}$, $J=8.4,1.1 \mathrm{~Hz}, 1 \mathrm{H}), 7.70(\mathrm{~d}, J=7.4 \mathrm{~Hz}, 1 \mathrm{H}), 7.57-7.53(\mathrm{~m}, 1 \mathrm{H})$, $7.45-7.41(\mathrm{~m}, 1 \mathrm{H}), 7.24(\mathrm{~d}, J=7.8 \mathrm{~Hz}, 2 \mathrm{H}), 6.91(\mathrm{~d}, J=8.0 \mathrm{~Hz}$, 1H), 5.24-5.17 (m, 1H, NCH), 4.09 (s, 3H, OMe), 2.30-2.21 (m, $2 \mathrm{H}, \beta-\mathrm{CH}_{2}$ ), 1.92-1.82 (m, 2H, $\left.\beta-\mathrm{CH}_{2}\right), 1.74\left(\mathrm{~s}, 3 \mathrm{H}, \mathrm{CH}_{3}\right), 1.36-$ $1.20\left(\mathrm{~m}, 16 \mathrm{H}, 8 \times \mathrm{CH}_{2}\right), 0.84(\mathrm{t}, J=6.5 \mathrm{~Hz}, 6 \mathrm{H}) .{ }^{13} \mathrm{C} \mathrm{NMR}\left(\mathrm{CDCl}_{3}\right.$, $150 \mathrm{MHz}): \delta / \mathrm{ppm}=165.62,164.67,156.42,149.90,134.47$, $132.86,132.51,132.22,131.60,130.85,130.12$, 128.70, 128.26, $127.00,126.83,125.54,123.58,122.88,122.36,121.64,121.23$, 102.85, 55.91, 54.67, 32.57, 31.93, 29.43, 27.14, 24.58, 22.78, 14.22. MS (EI, $70 \mathrm{eV}) \mathrm{m} / z$ (\%): 550 (22), 549 (50) [M $\left.\mathrm{M}^{+}\right], 380$ (6), 369 (13), 368 (62), 367 (100), 352 (8), 309 (5). HRMS (EI) for $\mathrm{C}_{37} \mathrm{H}_{43} \mathrm{NO}_{3}$ : calcd: 549.3243; found: 549.3238. EA for $\mathrm{C}_{37} \mathrm{H}_{43} \mathrm{NO}_{3}$ : calcd: C 80.84, N 2.55, H 7.88; found: C 80.32, N 2.54, H 8.00. $\mathrm{UV} / \mathrm{Vis}\left(\mathrm{CHCl}_{3}\right): \lambda_{\max }(\varepsilon)=329.0 \mathrm{~nm}(17100)$. Fluorescence $\left(\mathrm{CHCl}_{3}\right.$, $\left.\lambda_{\text {exc }}=329.0 \mathrm{~nm}\right): \lambda_{\max }=522.7 \mathrm{~nm}$. Fluorescence quantum yield $\left(\mathrm{CHCl}_{3}, \lambda_{\text {exc }}=329.0 \mathrm{~nm}, E_{329.0 \mathrm{~nm}, 1 \mathrm{~cm}}=0.063\right.$, reference: $N, N^{\prime}$-bis(tridecan-7-yl)perylene-3,4:9,10-tetracarboxylic diimide with $\Phi=1.00): ~ \Phi=0.40$.

\section{6-(4-(Trimethylsilyl)phenyl)-2-(tridecan-7-yl)-1H- benzo[de]isoquinoline-1,3(2H)-dione (3i)}

A Schlenk-flask was charged with 6-bromo-2-(tridecan-7-yl)$1 H$-benzo[de]isoquinoline-1,3(2H)-dione $(1.33 \mathrm{~g}, 2.92 \mathrm{mmol}$, 1.05 equiv.) and (4-(trimethylsilyl)phenyl)boronic acid (540 mg, $2.78 \mathrm{mmol}, 1.0$ equiv.). Both compounds were dissolved in toluene $(40 \mathrm{~mL})$ under a light argon-stream. $\mathrm{K}_{2} \mathrm{CO}_{3}(8.40 \mathrm{~g}$, $60.0 \mathrm{mmol}$ ) was dissolved in a mixture of water $(30 \mathrm{~mL})$ and EtOH $(5.00 \mathrm{~mL})$ and added to the Schlenk-flask followed by tetrakis(triphenylphosphine)palladium(0) (160 mg, $0.140 \mathrm{mmol}$, $5 \mathrm{~mol} \%$ ). The mixture was purged with argon for $30 \mathrm{~min}$ and then heated to $80{ }^{\circ} \mathrm{C}$. The reaction mixture was stirred at this temperature for further $17 \mathrm{~h}$. After cooling to room temperature, the organic phase was separated and the aqueous phase was extracted with toluene $(3 \times 30 \mathrm{~mL})$. Purification by column chromatography (silica, iso-hexane/ $\mathrm{CHCl}_{3}=1: 1$ ) gave compound $3 \mathbf{i}$ as green fluorescent oil $(1.19 \mathrm{~g}, 81 \%)$. IR (diamondATR, neat): $\tilde{\nu} / \mathrm{cm}^{-1}=2923,2855,1698,1656,1587,1455,1398$, 1349, 1237, 1179, 1111, 838, 820, 784, 761, 726, 695. ${ }^{1} \mathrm{H}$ NMR $\left(\mathrm{CDCl}_{3}, 400 \mathrm{MHz}\right): \delta / \mathrm{ppm}=8.71-8.56(\mathrm{br} \mathrm{s}, 2 \mathrm{H}), 8.30(\mathrm{dd}, J=8.5$, $1.1 \mathrm{~Hz}, 1 \mathrm{H}), 7.71(\mathrm{dd}, J=7.9,2.6 \mathrm{~Hz}, 3 \mathrm{H}), 7.51(\mathrm{~d}, J=8.1 \mathrm{~Hz}, 1 \mathrm{H})$, 5.28-5.16 (m, 1H, NCH), 2.35-2.22 (m, 2H, $\left.\beta-\mathrm{CH}_{2}\right), 1.93-1.79$ $\left(\mathrm{m}, 2 \mathrm{H}, \beta-\mathrm{CH}_{2}\right), 1.46-1.14\left(\mathrm{~m}, 16 \mathrm{H}, 8 \times \mathrm{CH}_{2}\right), 0.83(\mathrm{t}, J=6.9 \mathrm{~Hz}, 6 \mathrm{H})$,
0.36 (s, 9H, TMS). ${ }^{13} \mathrm{C} \mathrm{NMR}\left(\mathrm{CDCl}_{3}, 100 \mathrm{MHz}\right): \delta / \mathrm{ppm}=165.63$, 165.46, 164.58, 164.39, 146.65, 141.05, 139.33, 133.72, 132.44, 131.67, 131.34, 130.89, 130.58, 130.04, 129.29, 129.02, 127.91, $126.91,123.71,123.02,122.58,121.84,54.58,32.57,31.90$, $29.37,27.04,22.71,14.17,-0.97$. MS (EI, $70 \mathrm{eV}) \mathrm{m} / \mathrm{z}(\%)$ : 527 (20) $\left[\mathrm{M}^{+}\right], 348$ (8), 347 (29), 346 (100), 345 (26), 331 (11), 330 (33). HRMS (EI) for $\mathrm{C}_{34} \mathrm{H}_{45} \mathrm{NO}_{2} \mathrm{Si}$ : calcd: 527.3220; found: 535.3216. EA for $\mathrm{C}_{34} \mathrm{H}_{45} \mathrm{NO}_{2} \mathrm{Si}$ : calcd: $\mathrm{C} 77.37, \mathrm{~N} 2.65, \mathrm{H} 8.59$; found: $\mathrm{C} 77.02, \mathrm{~N} 2.73, \mathrm{H}$ 8.63. UV/Vis $\left(\mathrm{CHCl}_{3}\right): \lambda_{\max }(\varepsilon)=$ $356.0 \mathrm{~nm}$ (18600). Fluorescence $\left(\mathrm{CHCl}_{3}, \lambda_{\text {exc }}=356.0 \mathrm{~nm}\right)$ : $\lambda_{\text {max }}=425.4 \mathrm{~nm}$. Fluorescence quantum yield $\left(\mathrm{CHCl}_{3}, \lambda_{\text {exc }}=\right.$ $356.0 \mathrm{~nm}, E_{356.0 \mathrm{~nm}, 1 \mathrm{~cm}}=0.068$, reference: $N, N^{\prime}$-bis(tridecan7-yl)perylene-3,4:9,10-tetracarboxylic diimide with $\Phi=1.00)$ : $\Phi=0.79$.

\section{6-(4-Cyanophenyl)-2-(tridecan-7-yl)-1H-benzo[de]isoquinoline-} 1,3(2H)-dione $(3 \mathbf{j})$

Procedure A: Suzuki-coupling. A Schlenk-flask was charged with 6-bromo-2-(tridecan-7-yl)-1 $H$-benzo[de]isoquinoline-1,3(2H)dione (980 mg, $2.10 \mathrm{mmol}, 1.0$ equiv.) and 4-cyanophenylboronic acid (970 mg, $2.52 \mathrm{mmol}, 1.2$ equiv.). Both compounds were dissolved in toluene $(40 \mathrm{~mL})$ under a light argon stream. $\mathrm{K}_{2} \mathrm{CO}_{3}$ $(5.00 \mathrm{~g}, 36.2 \mathrm{mmol})$ was dissolved in a mixture of water $(20 \mathrm{~mL})$ and $\mathrm{EtOH}(8 \mathrm{~mL})$ and added to the Schlenk-flask followed by tetrakis-(triphenylphosphine)palladium(0) $(115 \mathrm{mg}, 0.110 \mathrm{mmol}$, $5.00 \mathrm{~mol} \%$ ). The mixture was purged with argon for $30 \mathrm{~min}$ and then heated to $80{ }^{\circ} \mathrm{C}$. The reaction mixture was stirred at this temperature for further $17 \mathrm{~h}$. After cooling to room temperature, the organic phase was separated and the aqueous phase was extracted with toluene $(3 \times 20 \mathrm{~mL})$. Purification by column chromatography (silica, iso-hexane $/ \mathrm{CHCl}_{3}=1: 1$ ) gave compound $3 \mathbf{j}$ as bright yellow oil (416 $\mathrm{mg}, 41 \%)$.

Procedure B: cyanation with $\mathrm{K}_{\mathbf{4}} \mathrm{Fe}(\mathrm{CN})_{6}$. A dry argon flushed Schlenk-flask was charged with potassium hexacyanidoferrate(II) $\left(\mathrm{K}_{4} \mathrm{Fe}(\mathrm{CN})_{6}, 128 \mathrm{mg}, 0.348 \mathrm{mmol}, 2.0\right.$ equiv.), potassium carbonate $\left(\mathrm{K}_{2} \mathrm{CO}_{3}, 72.1 \mathrm{mg}, 0.522 \mathrm{mmol}, 3.0\right.$ equiv.) and [1,1'-bis(diphenylphosphino)ferrocene]dichloropalladium(II) ( $\mathrm{Pd}(\mathrm{dppf}) \mathrm{Cl}_{2}, 12.7 \mathrm{mg}$, $0.0174 \mathrm{mmol}, 10 \mathrm{~mol} \%)$. A solution of 6-(4-iodophenyl)-2(tridecan-7-yl)-1H-benzo-[de]isoquinoline-1,3(2H)-dione $(101 \mathrm{mg}$, $0.174 \mathrm{mmol}$ ) in $1.7 \mathrm{~mL}$ of dry $N, N$-dimethylformamide was added at room temperature under argon. The reaction mixture was stirred at $100{ }^{\circ} \mathrm{C}$ for $4 \mathrm{~h}$, allowed to cool down and the solvent was removed under reduced pressure. The crude residue was treated with $15 \mathrm{~mL}$ of sat. aq. $\mathrm{NH}_{4} \mathrm{Cl}$ solution and extracted with chloroform $(3 \times 15 \mathrm{~mL})$. Purification by column chromatography (silica, iso-hexane/ $\mathrm{CHCl}_{3}=1: 1$ ) gave compound $\mathbf{3 j}$ as bright yellow oil (54.9 mg, $0.114 \mathrm{mmol}, 66 \%$ ). IR (diamond-ATR, neat): $\tilde{\nu} / \mathrm{cm}^{-1}=2923,2855,1699,1656,1588,1465,1397,1349$, 1327, 1238, 1179, 1103, 844, 784, 759. ${ }^{1} \mathrm{H} \mathrm{NMR}\left(\mathrm{CDCl}_{3}, 600 \mathrm{MHz}\right)$ : $\delta / \mathrm{ppm}=8.71-8.60(\mathrm{br} \mathrm{s}, 2 \mathrm{H}), 8.36(\mathrm{dd}, J=8.5 \mathrm{~Hz}, 1.0 \mathrm{~Hz}, 1 \mathrm{H}), 7.89$ $(\mathrm{d}, J=8.4 \mathrm{~Hz}, 2 \mathrm{H}), 7.78(\mathrm{~d}, J=7.5 \mathrm{~Hz}, 1 \mathrm{H}), 7.77-7.73(\mathrm{~m}, 1 \mathrm{H}), 7.67$ $(\mathrm{d}, J=8.4 \mathrm{~Hz}, 2 \mathrm{H}), 5.23-5.18(\mathrm{~m}, 1 \mathrm{H}, \mathrm{NCH}), 2.30-2.23(\mathrm{~m}, 2 \mathrm{H}$, $\left.\beta-\mathrm{CH}_{2}\right), 1.89-1.82\left(\mathrm{~m}, 2 \mathrm{H}, \beta-\mathrm{CH}_{2}\right), 1.37-1.20\left(\mathrm{~m}, 16 \mathrm{H}, 8 \times \mathrm{CH}_{2}\right)$, $0.84(\mathrm{t}, J=7.1 \mathrm{~Hz}, 6 \mathrm{H}) .{ }^{13} \mathrm{C} \mathrm{NMR}\left(\mathrm{CDCl}_{3}, 150 \mathrm{MHz}\right): \delta / \mathrm{ppm}=$ $165.67,165.49,164.58,164.41$, 146.08, 140.64, 138.49, 132.32, $131.81,131.40,131.02,130.70,130.08,129.10,128.02,127.56$, 
$127.12,123.83,123.10,122.77,122.05,54.70,32.60,31.94$, 29.41, 27.09, 22.76, 14.21. MS (EI, $70 \mathrm{eV}) \mathrm{m} / z$ (\%): 480 (2) $\left[\mathrm{M}^{+}\right]$, 299 (17). HRMS (EI) for $\mathrm{C}_{32} \mathrm{H}_{36} \mathrm{~N}_{2} \mathrm{O}_{2}$ : calcd: 480.2777; found: 480.2773. UV/Vis $\left(\mathrm{CHCl}_{3}\right): \lambda_{\max }(\varepsilon)=366.4 \mathrm{~nm}(23700)$. Fluorescence $\left(\mathrm{CHCl}_{3}, \lambda_{\text {exc }}=366.4 \mathrm{~nm}\right): \lambda_{\text {max }}=447.4 \mathrm{~nm}$. Fluorescence quantum yield $\left(\mathrm{CHCl}_{3}, \lambda_{\text {exc }}=366.4 \mathrm{~nm}, E_{366.4 \mathrm{~nm}, 1 \mathrm{~cm}}=0.073\right.$, reference $N, N^{\prime}$-bis(tridecan-7-yl)perylene-3,4:9,10-tetracarboxylic diimide with $\Phi=1.00): \Phi=0.54$.

\section{6-(4-Iodophenyl)-2-(tridecan-7-yl)-1H-benzo[de]isoquinoline- 1,3(2H)-dione (3k)}

A dry, argon flushed Schlenk-flask was charged with 6-(4(trimethylsilyl)phenyl)-2-(tridecan-7-yl)-1H-benzo[de]iso-quinoline1,3(2H)-dione ( $840 \mathrm{mg}, 1.60 \mathrm{mmol}, 1.0$ equiv.) and dry dichloromethane $(3.5 \mathrm{~mL})$. The flask was cooled to $0{ }^{\circ} \mathrm{C}$ and iodine monochloride (260 mg, $1.60 \mathrm{mmol}, 1.0$ equiv.) was added dropwise to the mixture. After stirring at $0{ }^{\circ} \mathrm{C}$ for $10 \mathrm{~min}$, the reaction mixture was quenched with sat. aq. $\mathrm{Na}_{2} \mathrm{~S}_{2} \mathrm{O}_{3}$ solution $(5 \mathrm{~mL})$, extracted with dichloromethane $(3 \times 10 \mathrm{~mL})$ and dried over anhydrous $\mathrm{MgSO}_{4}$. After filtration, the solvents were evaporated in vacuo. The crude product was purified by flash column chromatography (silica, $\mathrm{CHCl}_{3}$ ) to obtain $3 \mathbf{k}$ as yellow oil $(770 \mathrm{mg}$, 83\%). IR (diamond-ATR, neat): $\tilde{\nu} / \mathrm{cm}^{-1}=2922,2854,1698,1656$, 1588, 1487, 1463, 1398, 1349, 1324, 1238, 1178, 1101, 1005, 821, 784, 758. ${ }^{1} \mathrm{H} \mathrm{NMR}\left(\mathrm{CDCl}_{3}, 600 \mathrm{MHz}\right): \delta / \mathrm{ppm}=8.65-8.56(\mathrm{~m}, 2 \mathrm{H})$, $8.18(\mathrm{~d}, J=8.4 \mathrm{~Hz}, 1 \mathrm{H}), 7.85(\mathrm{~d}, J=8.4 \mathrm{~Hz}, 2 \mathrm{H}), 7.70-7.66(\mathrm{~m}, 1 \mathrm{H})$, 7.64 (d, $J=7.5 \mathrm{~Hz}, 1 \mathrm{H}), 7.23$ (d, $J=8.3 \mathrm{~Hz}, 2 \mathrm{H}), 5.22-5.15(\mathrm{~m}, 1 \mathrm{H}$, $\mathrm{NCH}), 2.27-2.20\left(\mathrm{~m}, 2 \mathrm{H}, \beta-\mathrm{CH}_{2}\right), 1.86-1.79\left(\mathrm{~m}, 2 \mathrm{H}, \beta-\mathrm{CH}_{2}\right), 1.32-$ $1.15\left(\mathrm{~m}, 16 \mathrm{H}, 8 \times \mathrm{CH}_{2}\right), 0.79(\mathrm{t}, J=7.1 \mathrm{~Hz}, 6 \mathrm{H}) .{ }^{13} \mathrm{C} \mathrm{NMR}\left(\mathrm{CDCl}_{3}\right.$, $150 \mathrm{MHz}): \delta / \mathrm{ppm}=165.41,165.22,164.35,164.13,145.17$, 138.40, 137.92, 133.52, 131.82, 131.65, 131.19, 130.97, 130.44, 129.71, 128.92, 127.76, 127.15, 126.97, 123.74, 123.03, 122.87, 122.19, 94.61, 54.57, 32.48, 31.84, 29.30, 26.98, 22.65, 14.13. MS

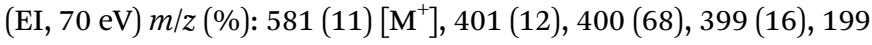
(12), 198 (100). HRMS (EI) for $\mathrm{C}_{31} \mathrm{H}_{36} \mathrm{INO}_{2}$ : calcd: 581.1791; found: 581.1781. EA for $\mathrm{C}_{31} \mathrm{H}_{36} \mathrm{INO}_{2}$ : calcd: $\mathrm{C}$ 64.03, $\mathrm{N} 2.41$, H 6.24; found: C 63.88, N 2.49, H 6.32.

\section{6-(4'-Methoxy-[1,1'-biphenyl]-4-yl)-2-(tridecan-7-yl)-1H- benzo[de]isoquinoline-1,3(2H)-dione (31)}

A dry argon flushed Schlenk-flask was charged with 4-iodoanisole (40.0 mg, $0.16 \mathrm{mmol}, 1.0$ equiv.) and dry THF (1.00 mL). An iodine/magnesium exchange was performed using iPrMgCl$\cdot \mathrm{LiCl}$ ( $0.13 \mathrm{~mL}, 0.16 \mathrm{mmol}, 1.26 \mathrm{M}, 1.0$ equiv.) within $20 \mathrm{~min}$ at $0{ }^{\circ} \mathrm{C}$, followed by transmetalation with $\mathrm{ZnCl}_{2}(0.18 \mathrm{~mL}, 0.18 \mathrm{mmol}$, $1.00 \mathrm{M}, 1.1$ equiv.). The freshly prepared zinc species was transferred via a syringe to another argon flushed Schlenk-flask which was previously charged with 6-(4-iodophenyl)-2-(tridecan-7-yl)-1 $H$-benzo[de]isoquinoline-1,3-(2H)-dione (96.0 mg, $0.16 \mathrm{mmol}, 1.0$ equiv.), palladium(0)bis-(dibenzylideneacetone) (5 mg, $0.008 \mathrm{mmol}$, $5 \mathrm{~mol} \%$ ), tris-(2-furyl)phosphine (4.0 mg, $0.016 \mathrm{mmol}, 10 \mathrm{~mol} \%$ ) and dry THF $(3 \mathrm{~mL})$. The reaction mixture was stirred at $50{ }^{\circ} \mathrm{C}$ for $12 \mathrm{~h}$, quenched with sat. aq. $\mathrm{NH}_{4} \mathrm{Cl}$ solution, extracted with dichloromethane $(3 \times 5 \mathrm{~mL})$ and dried over $\mathrm{MgSO}_{4}$. After filtration, the solvents were evaporated in vacuo. The crude product was purified by flash column chromatography (silica, iso-hexane/dichloromethane $=1: 1$ ) to obtain 31 as a yellow solid (80.0 mg, 89\%). M.p.: $104{ }^{\circ} \mathrm{C}$. IR (diamond-ATR, neat): $\tilde{\nu} / \mathrm{cm}^{-1}=2924,2854,1697,1652,1604,1587,1497,1464$, 1396, 1349, 1288, 1238, 1177, 1106, 1039, 823, 784, 760. ${ }^{1} \mathrm{H}$ NMR $\left(\mathrm{CDCl}_{3}, 600 \mathrm{MHz}\right): \delta / \mathrm{ppm}=8.70-8.56(\mathrm{~m}, 2 \mathrm{H}), 8.35$ (dd, $J=8.5,1.1 \mathrm{~Hz}, 1 \mathrm{H}), 7.76-7.73(\mathrm{~m}, 3 \mathrm{H}), 7.73-7.69(\mathrm{~m}, 1 \mathrm{H})$, $7.64(\mathrm{~d}, J=8.9 \mathrm{~Hz}, 2 \mathrm{H}), 7.57$ (d, $J=8.4 \mathrm{~Hz}, 2 \mathrm{H}), 7.04$ (d, $J=8.8$ $\mathrm{Hz}, 2 \mathrm{H}), 5.23(\mathrm{~m}, 1 \mathrm{H}, \mathrm{NCH}), 7.04$ (s, 3H, OMe), 2.31-2.21 (m, $\left.2 \mathrm{H}, \beta-\mathrm{CH}_{2}\right), 1.89-1.80\left(\mathrm{~m}, 2 \mathrm{H}, \beta-\mathrm{CH}_{2}\right), 1.36-1.20(\mathrm{~m}, 16 \mathrm{H}$, $\left.8 \times \mathrm{CH}_{2}\right), 0.83(\mathrm{t}, J=7.1 \mathrm{~Hz}, 6 \mathrm{H}) .{ }^{13} \mathrm{C} \mathrm{NMR}\left(\mathrm{CDCl}_{3}, 150 \mathrm{MHz}\right)$ : $\delta / \mathrm{ppm}=165.70,164.46,159.69,146.41,141.13,137.31,132.94$, $132.47,131.74,131.41,130.96,130.63,130.48,130.11,129.08$, 128.34, 127.95, 127.04, 127.00, 123.76, 123.04, 122.54, 121.82, $114.57,55.55,54.65,32.60,31.93,29.41,27.08,22.75,14.21 . \mathrm{MS}$

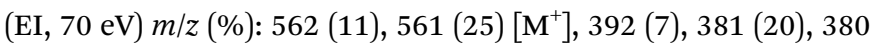
(76), 379 (100), 364 (7), 336 (7). HRMS (EI) for $\mathrm{C}_{38} \mathrm{H}_{43} \mathrm{NO}_{3}$ : calcd: 561.3243; found: 561. 3229. EA for $\mathrm{C}_{38} \mathrm{H}_{43} \mathrm{NO}_{3}$ : calcd: $\mathrm{C} 81.25, \mathrm{~N}$ 2.49, H 7.72; found: $\mathrm{C} 81.37, \mathrm{~N} 2.49, \mathrm{H}$ 7.76. UV/Vis $\left(\mathrm{CHCl}_{3}\right)$ : $\lambda_{\text {max }}(\varepsilon)=364.4 \mathrm{~nm}(27500)$. Fluorescence $\left(\mathrm{CHCl}_{3}, \lambda_{\text {exc }}=364.4 \mathrm{~nm}\right)$ : $\lambda_{\text {max }}=492.2 \mathrm{~nm}$. Fluorescence quantum yield $\left(\mathrm{CHCl}_{3}, \lambda_{\text {exc }}=\right.$ $364.4 \mathrm{~nm}, E_{364.4 \mathrm{~nm}, 1 \mathrm{~cm}}=0.053$, reference: $N, N^{\prime}$-bis(tridecan-7yl)perylene-3,4:9,10-tetracarboxylic diimide with $\Phi=1.00$ ): $\Phi=0.67$.

\section{Notes and references}

1 (a) J. Malinge, C. Allain, A. Brosseau and P. Audebert, Angew. Chem., Int. Ed., 2012, 51, 8534; (b) A. Von Dorlars, C.-W. Schellhammer and J. Schroeder, Angew. Chem., Int. Ed. Engl., 1975, 14, 665; (c) L.-B. Li, S.-J. Ji and Y. Liu, Chin. J. Chem., 2008, 26, 595; (d) C. D. Geddes and J. R. Lakowicz, Reviews in Fluorescence, 2004; (e) L. D. Patsenker and Y. Y. Artyukhova, J. Mol. Struct., 2003, 655, 311; $(f)$ J. Liu, G. Tu, Q. Zhou, Y. Cheng, Y. Geng, L. Wang, D. Ma, X. Jing and F. Wang, J. Mater. Chem., 2006, 16, 1431; $(g)$ L. Dai, D. Wu, Q. Qiao, W. Yin, J. Yin and Z. Xu, Chem. Commun., 2016, 52, 2095; (h) Y. Liu, H. Ma, L. Zhang, Y. Cui, X. Liu and J. Fang, Chem. Commun., 2016, 52, 2296.

2 J. Liu, G. Tu, Q. Zhou, Y. Cheng, Y. Geng, L. Wang, D. Ma, X. Jing and F. Wang, Adv. Mater., 2007, 19, 531.

3 (a) Brit. Pat., GB 402309, I. G. Farbenindustrie AG, 1933; Chem. Abstr., 1934, 28, 27267; (b) W. K. Koerber and F. L. Schouteden, Ger. Pat., DE 1108560, Gevaert Photo-Producten N.V., 1957 (Chem. Abstr. 1962, 56, 32447); (c) B. M. Krasovitskii, D. G. Pereyaslova, E. G. Yushko and G. V. Tatsii, Monokrist., Stsintill. Org. Lyuminofory, 1967, 1, 92; Chem. Abstr. 1968, 69, 60030.

4 (a) W. Bradley and F. W. Pexton, J. Chem. Soc., 1954, 4432; (b) I. Grabtchev and Ts. Philipova, Dyes Pigm., 1995, 27, 321; (c) A. Pardo, J. M. L. Poyato, E. Martin, J. J. Camacho, D. Reyman, M. F. Brana and J. M. Castellano, J. Photochem. Photobiol., A, 1989, 46, 323; (d) V. Wintgens, P. Valat, J. Kossanyi, A. Demeter, L. Biczok and T. Berces, New J. Chem., 1996, 20, 1149; (e) X. Qian, Y. Zhang, K. Chen, Z. Tao and Y. Shen, Dyes Pigm., 1996, 32, 229; $(f)$ G. J.-F. Demets, E. R. Triboni, E. B. Alvarez, G. M. Arantes, 
P. B. Filho and M. J. Politi, Spectrochim. Acta, Part A, 2006, 63, 220.

5 (a) R. Umeda, H. Nishida, M. Otono and Y. Nishiyama, Tetrahedron Lett., 2011, 52, 5494; (b) S. Dhar, S. S. Roy, D. K. Rana, S. Bhattacharya, S. Bhattacharya and S. C. Bhattacharya, J. Phys. Chem. A, 2011, 115, 2216.

6 (a) I. A. Zhmyreva, V. V. Zelinskii, V. P. Kolobkov and N. D. Krasnitskaya, Dokl. Akad. Nauk SSSR, 1959, 129, 1089 (Chem. Abstr., 1961, 55, 141336); (b) K. Schwetlick, Kinetic methods for studying reaction mechanisms, VEB Deut. Verlag Wiss., Berlin, 1971, p. 165 (Chem. Abstr. 1972, 77, 79990); (c) C. Reichardt and T. Welton, Solvents and Solvent Effects in Organic Chemistry, Wiley-VCH, Weinheim, 4th edn, 2011, ISBN 3-527-26805-7.

7 (a) L. Wang, J. Liu, Y. Cheng and Y. Geng, Faming Zhuanli Shenqing, CN 1637112, 2005 (Chem. Abstr. 2006, 144, 180451); (b) X. Fang, M. Guo, X. Yuan and M. Lin, Faming Zhuanli Shenqing, CN 103923008, 2014 (Chem. Abstr. 2014, 161, 275799); (c) X. Huang, Y. Fang, X. Li, Y. Xie and W. Zhu, Dyes Pigm., 2011, 90, 297.

8 (a) H. Langhals, Chromophores for picoscale optical computers, in Fundamentals of picoscience, ed. K. Sattler, Taylor \& Francis Inc. CRC Press Inc., Bosa Roca/US, 2013, p. 705; ISBN 13: 9781466505094, ISBN 10: 1466505095; $(b)$ H. Langhals, Helv. Chim. Acta, 2005, 88, 1309.

9 (a) D. Haas, J. M. Hammann, R. Greiner and P. Knochel, ACS Catal., 2016, 6, 1540; (b) E.-i. Negishi, L. F. Valente and M. Kobayashi, J. Am. Chem. Soc., 1980, 102, 3298; (c) E.-i. Negishi, Acc. Chem. Res., 1982, 15, 340; (d) A. de Meijere and F. Diederich, Metal-Catalyzed Cross-Coupling Reactions, Wiley-VCH, Weinheim, 2004.

10 (a) N. Barl, V. Werner, C. Saemann and P. Knochel, Heterocycles, 2014, 88, 827; (b) A. Krasovskiy and P. Knochel, Angew. Chem., Int. Ed., 2004, 43, 3333.
11 (a) K. Dimroth, C. Reichardt, T. Siepmann and F. Bohlmann, Liebigs Ann. Chem., 1963, 661, 1; (b) C. Reichardt, Angew. Chem., Int. Ed., 1965, 4, 29; (c) C. Reichardt and R. Müller, Liebigs Ann. Chem., 1976, 1937; (d) C. Reichardt, Pure Appl. Chem., 2008, 80, 1415; (e) V. G. Machado, R. I. Stock and C. Reichardt, Chem. Rev., 2014, 114, 10429; $(f)$ J. P. Cerón-Carrasco, D. Jacquemin, C. Laurence, A. Planchat, C. Reichardt and K. Sraïdi, J. Phys. Org. Chem., 2014, 27, 512; $(g)$ M. Afri, H. E. Gottlieb and A. A. Frimer, Can. J. Chem., 2014, 92, 128-134; (h) T. Etienne, C. Michaux, A. Monari, X. Assfeld and E. A. Perpète, Dyes Pigm., 2014, 100, 24.

12 A. Kawski, P. Bojarski and B. Kukliński, Chem. Phys. Lett., 2008, 463, 410.

13 J.-L. M. Abboud, R. W. Taft and M. J. Kamlet, J. Chem. Soc., Perkin Trans. 2, 1985, 815.

14 J. Catalán, J. Phys. Chem. B, 2009, 113, 5951.

15 L. G. S. Brooker, A. C. Craig, D. W. Heseltine, P. W. Jenkins and L. L. Lincoln, J. Am. Chem. Soc., 1965, 87, 2443.

16 Correlation Analysis in Chemistry - Recent Advances, ed. N. B. Chapman and J. Shorter, Plenum Press, New York, London, 1978, ISBN 0-306-31068-6.

17 C. Cao, X. Liu, Q. Qiao, M. Zhao, W. Yin, D. Mao, H. Zhang and Z. Xu, Chem. Commun., 2014, 50, 15811.

18 (a) W. Rettig, Angew. Chem., Int. Ed., 1986, 25, 971; (b) D. Oesch and N. W. Luedtke, Chem. Commun., 2015, 51, 12641; (c) Z. H. Guo, Z.-X. Jin, J.-Y. Wang and J. Pei, Chem. Commun., 2014, 50, 6088; (d) S. Chevreux, R. Paulino Neto, C. Allain, K. Nakatani, P. Jacques, I. Ciofini and G. Lemercier, Phys. Chem. Chem. Phys., 2015, 17, 7639; (e) R. Ghosh, A. Nandi and D. K. Palit, Phys. Chem. Chem. Phys., 2016, 18, 7661; $(f)$ H. Tanaka, K. Shizu, H. Nakanotani and C. Adachi, Chem. Mater., 2013, 25, 3766 . 